\title{
Gene Expression Analysis for Statin-induced Cytotoxicity from Rat Primary Hepatocytes
}

\author{
Moon-Jeong Ko, Joon-Ik Ahn, Hee Jung Shin, \\ Hye Soo Kim, Hye Joo Chung and Ho-Sang \\ Jeong*
}

National Institution of Food and Drug Safety Evaluation, Seoul 122-704, Korea, Korea Food and Drug Administration, Seoul 122-704, Korea

\begin{abstract}
Statins are competitive inhibitors of hydroxy-3-methyl glutaryl coenzyme A (HMG-CoA) reductase and used most frequently to reduce plasma cholesterol levels and to decrease cardiovascular events. However, statins also have been reported to have undesirable side effects such as myotoxicity and hepatotoxicity associated with their intrinsic efficacy mechanisms. Clinical studies recurrently reported that statin therapy elevated the level of liver enzymes such as ALT and AST in patients suggesting possible liver toxicity due to statins. This observation has been drawn great attention since statins are the most prescribed drugs and statin-therapy was extended to a larger number of high-risk patients. Here we employed rat primary hepatocytes and microarray technique to understand underlying mechanism responsible for statin-induced liver toxicity on cell level. We isolated genes whose expressions were commonly modulated by statin treatments and examined their biological functions. It is of interest that those genes have function related to response to stress in particular immunity and defense in cells. Our study provided the basic information on cellular mechanism of statin-induced cytotoxicity and may serve for finding indicator genes of statin -induced toxicity in rat primary hepatocytes.
\end{abstract}

Keywords: cytotoxicity, gene expression, hepatocyte, microarray, statin

\section{Introduction}

Statins are competitive hydroxy-3-methyl glutaryl coenzyme A (HMG-CoA) reductase inhibitors that inhibit the synthesis of cholesterol from mevalonic acid and the drugs most frequently used to reduce plasma cholester-

*Corresponding author: E-mail hosa33@korea.kr

Tel +82-2-380-1804, Fax +82-2-388-6457

Accepted 2 March 2010 ol levels and decrease cardiovascular events (Abu et al., 2005). However, the side effects associated with the use of statins have been highlighted by the withdrawal of cerivastatin from the market in 2001 (Baycol website) due to its causing a relatively high incidence of myopathy and rhabdomyolysis (Yamazaki et al., 2006).

Together with myotoxicity, there have been lingering concerns regarding statin-induced hepatotoxicity from the time of introduction of lovastatin in 1987, the first member of the class. Millions of patients have now received these drugs. Asymptomatic increases in aminotransferase levels develop frequently (Maddrey et al., 2005). Since statins inhibit cholesterol synthesis in the liver much more than in any other tissue, it is not surprising that elevations in the liver enzymes ALT and AST have been noted since the first clinical trial with lovastatin (Hamelin et al., 1998). In the case of statin therapy, four types of hepatic syndromes need to be considered; acute liver failure, hepatitis, cholestasis and transaminitis. Initial toxicology studies in animals suggested that statins may cause significant liver problems. Although the usual doses of lovastatin did not cause significant liver injury, when given in very high dose they caused hepatocellular necrosis in rabbits (Chalasani, et al., 2005). Similarly, high doses of simvastatin caused hepatocellular necrosis in guinea pig (Chalasani et al., 2005). The liver injury in these animals could be prevented or reversed with supplementing animals with mevalonate suggesting that depletion of mevalonate or its down-stream metabolite might be responsible for liver injury (Chalasani et al., 2005).

According to adverse effect reports, it seemed that all of these hepatic adverse effects do not have any significant increase with statin therapy (Abu, et al., 2005). However, as the use of lipid lowering treatment including statins was extended to a larger number of high-risk patients, the potential of statin-induced liver toxicity became a major concern for the safety profile of statins. Moreover, a large-scale clinical study encouraged the use of statins in a wide range of patients at varying levels of risk for cardiovascular events and independently of baseline cholesterol levels (Heart Protection Study, 2002, Wilmshurst et al., 2002).

Taken together, it seemed that the most concerning issue for statin safety resides its potential to cause acute liver failure during wide use of cholesterol lowering therapy. To address this concern, it would be helpful to understand underlying mechanism of statin-in- 
duced toxicity using a commonly used system. In the presented work, rat primary hepatocytes were used as a model system to assess statin-induced toxicity by combining microarray technique. This effort will provide deeper knowledge of the mechanisms responsible for the toxic effects of statins along with putative indicator genes for statin -induced toxicity in rat primary hepatocytes.

\section{Methods}

\section{Preparation of rat primary hepatocytes}

Rat primary hepatocytes were isolated using a modified method described in elsewhere (Klaunig, et al.) and by Percoll centrifugation. All media and reagents were purchased from Gibco (NY, USA) unless mentioned differently. In brief, liver was perfused via portal vein first with $\mathrm{Ca}^{2+} / \mathrm{Mg}^{2+}$ free Hank's balanced salt solution (HBSS-) containing $0.5 \mathrm{mM}$ EGTA and $10 \mathrm{mM}$ HEPES for $2 \sim 3 \mathrm{~min}$ and then with HBSS with $\mathrm{Ca}^{2+} / \mathrm{Mg}^{2+}$ containing $10 \mathrm{mM}$ HEPES and $0.5 \mathrm{mg} / \mathrm{ml}$ collagenase (Wako) for 6 7 $\mathrm{min}$ at a flow rate of $20 \mathrm{ml} / \mathrm{min}$. Perfused livers were isolated, decapsulated and dispersed in ice-cold HBSS-. Dispersed cells were filtered with $100 \mu \mathrm{m}$ meshes, rinsed and suspended in ice-cold $50 \%(\mathrm{v} / \mathrm{v})$ Percoll (Amersham, NJ, USA) and centrifuged at $50 \mathrm{~g}$ for $10 \mathrm{~min}$ at $4^{\circ} \mathrm{C}$. Hepatocytes were rinsed and suspended in Williams' $E$ culture media supplemented with $10 \%$ (v/v) FCS, 2 mM L-glutamate, $100 \mathrm{U} / \mathrm{ml}$ penicillin, $100 \mu \mathrm{g} / \mathrm{ml}$ streptomycin and $10 \mathrm{mM}$ HEPES. Cell cultures showing more than $80 \%$ viability, based on trypan blue exclusion, were used for further experiment. All cultures were maintained at $37^{\circ} \mathrm{C}$ in a water-saturated $5 \% \mathrm{CO}_{2}$ incubator and culture media were changed every day.

\section{Cell viability assay through MTT assay}

Resazurin assay was performed to estimate cell toxicity of each drug using rat primary hepatocytes. Drugs were first dissolved in DMSO (Sigma), diluted to the desired concentration with water and administrated to cells for 24 hours. Then $20 \mu \mathrm{L}$ of CellTiter-Blue (Promega) were added and the treated cells were cultivated in $\mathrm{CO}_{2}$ incubator (NuAire, US/NU-4350G) for 4 hours. The level of toxicity was observed by optical density using microplate reader (SpectraMax plus/384, Molecular Devices, USA) at wavelength of $570 \mathrm{~nm}$.

\section{Drug treatment and RNA extraction}

The analytic grade statins were purchased from Sequoia
Research Product (Pangbourne, UK). The cells were treated with the drugs at designated concentrations (EC, $1 / 25$ of $\mathrm{LC}_{20}, 1 / 5$ of $\mathrm{LC}_{20}$ and $\mathrm{LC}_{20}$ ) for 24 hours and lysed with lysis buffer (Invitrogen). The RNAs were extracted using easy-spin ${ }^{T M}$ Total RNA Extraction Kit (Invitrogen). Extracted RNAs were quantified with spectrophotometer (NanoDrop ND-1000, Rockland, DE, USA), and their purity was estimated by ratio of optical density at $230 / 260 \mathrm{~nm}$ and $260 / 280 \mathrm{~nm}$ of wavelength.

\section{Analysis of gene expression using DNA micro- array}

Rat Genome 230 2.0 GeneChips (Affymetrix, USA) were used to profile gene expression modulation caused by statins in this experiment. All procedures were followed by the manufacturer's protocol described in elsewhere (Ahn et al., 2009). In short, the first and second strand cDNAs were synthesized using One-Cycle cDNA Synthesis Kit 1 and Sample Cleanup Module 1. cRNAs were transcribed from the synthesized cDNA by In Vitro Transcription (IVT) Labeling Kit 1, and fragmented in $5 \mathrm{X}$ fragmentation buffer at $94^{\circ} \mathrm{C}$ for $35 \mathrm{~min}$. The fragmented cRNAs were hybridized in the gene chips using Hybridization Control Kit 1 , and incubated at $45^{\circ} \mathrm{C}$ for 16 hours. Then the chips were washed and scanned using Affymetrix GeneChip Scanner.

\section{Micorarray data analysess}

The microarray data were uploaded to Genplex 3.0 program (Istech, Korea) and normalized with MAS5, Global \& scale method recommended by Affymetrix. Further statistical analyses were mainly performed using tools provided by Genplex program.

\section{Results and Discussion}

\section{Cell viability assay and determination of $\mathrm{LC}_{20}$ for statins}

We treated rat primary hepatocytes with variety of concentration of test compounds, cerivastatin, simvastatin and lovastatin for $24 \mathrm{~h}$ to monitor their effects on cell viability using MTT assay. Cerivastatin, simvastatin and lovastatin showed significant cell death and revealed very typical viability curves with dose-dependent manner (Fig. 1). The LC20 for each drug were determined as $160 \mu \mathrm{M}, 120 \mu \mathrm{M}$ and $200 \mu \mathrm{M}$ respectively. Cell viability test revealed that simvastatin caused more cytotoxicity than cerivastatin and lovastatin in rat primary hepatocytes. This observation was further confirmed by other cell viability assay using HepG2 cells (data not shown) 

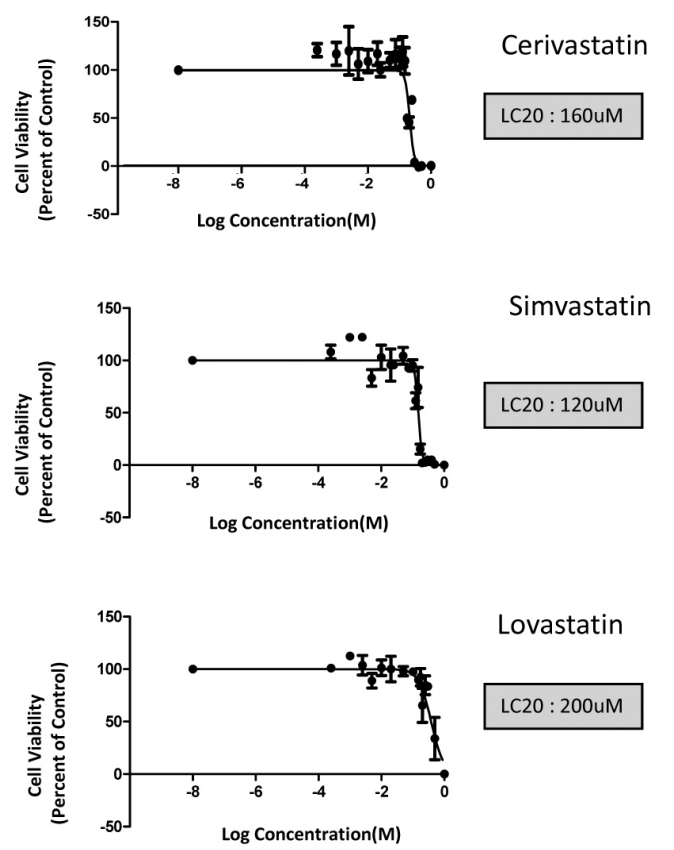

Fig. 1. Cytotoxicity assay results from rat primary hepatocytes treated with statins. Cells were incubated with the indicated concentrations of drugs for 24 hour, followed by cytotoxicity assay. Data are expressed as percentage of drug untreated cells and error bars represent standard error of the mean. Cerivastatin, Simavastatin and lovastatin showed comparable $\mathrm{LC}_{20}$ as $160 \mu \mathrm{M}, 120 \mu \mathrm{M}$ and $200 \mu \mathrm{M}$ respectively. where simvastatin also showed higher cytotoxicity than other two statins. These in vitro observations could not explain or reflect exactly what happens in clinical use of statins but still may arouse a question concerning liver toxicity induced by high dose of statins (Bolego, et al., 2002). Based on the cell viability test and bibliographical searches, the concentrations of drugs for the experiment were set at $1 / 25$ fold of $L_{2}, 1 / 5$ fold of $L_{20}$ and $L_{2} C_{20}$. An additional concentration $(0.5 \mu \mathrm{M})$ was added to the experiment to monitor the expression of genes which are related to the drug efficacy.

\section{Analysis of gene expression from rat primary hepatocytes treated by statins}

Using four different drug concentrations and an untreated control, the effect of statins on gene expression in rat primary hepatocytes was assessed by microarray experiments. Gene expression pattern of each drug was analyzed by One-way ANOVA ( $p$ value $<0.05$ ) with multiple test correction (Benjamini-Hochberg FDR) and showed 6,923, 2,688 and 4,383 genes significantly modulated at least one dose point of cerivastatin, lovastatin and simvastatin treatment, respectively. To examine relations between drug concentration and expression pattern, hierarchical clustering analysis (HCA) was performed for each drug treatment (Fig. 2). The HCA revealed grouping of gene expression profiles along with

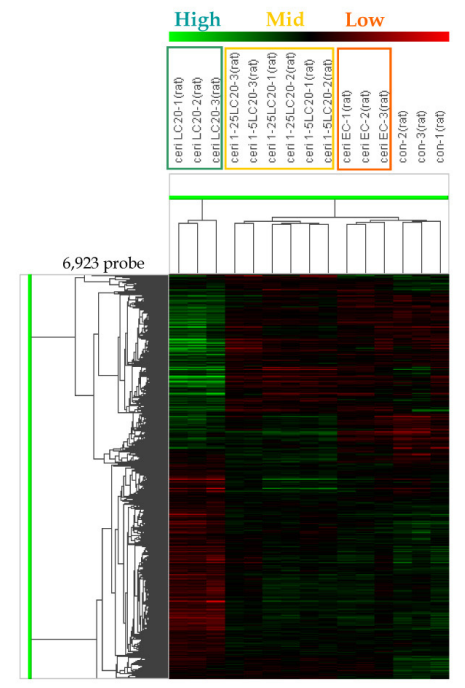

Cerivastatin

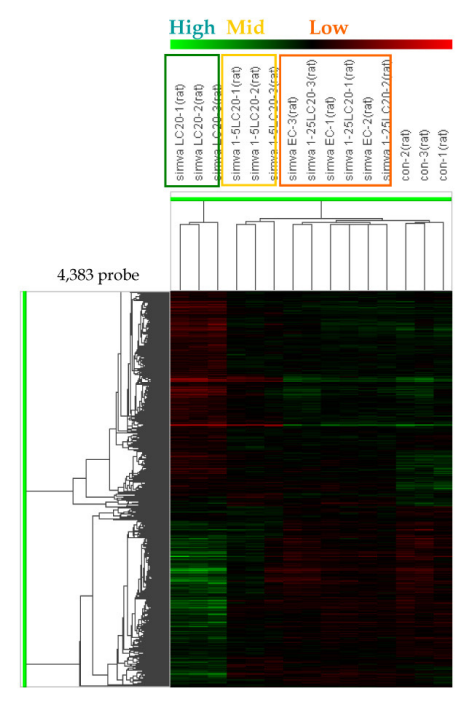

Simvastatin

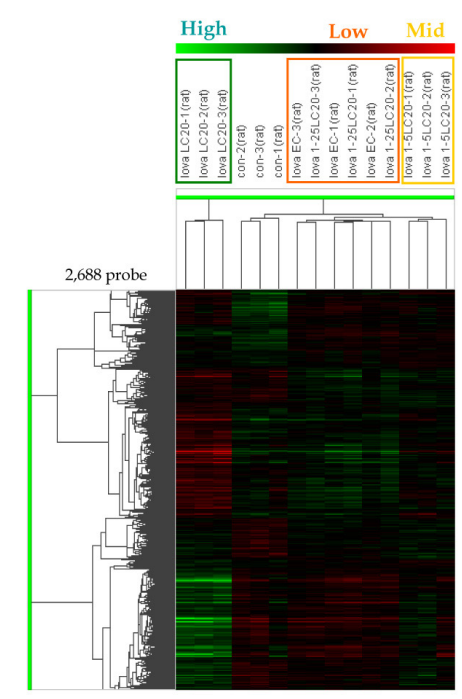

Lovastatin

Fig. 2. Hierarchical clustering analysis of differentially expression gene patterns in statin-treated rat primary hepatocytes. The probes sets used for the analysis were selected using one way ANOVA test ( $p$ value $<0.05$ ). HCA clustered two near dose groups into the same cluster. In cerivastatin treated samples, 1/25 and 1/5 $\mathrm{LC}_{20}$ dose groups were clustered into the same group unlikely simvastatin and lovastatin treated samples which showed clustering of EC and $1 / 25$ LC $_{20}$ dose groups into the same group. 
the drug concentrations and resulted in four distinctive hierarchical clusters in samples instead of five clusters due to grouping of $1 / 25$ and $1 / 5 L_{20}$ samples in the same cluster. Simvastatin and lovastatin treatment resulted in grouping EC and 1/25 LC $_{20}$ samples into the same cluster whereas cerivastatin treatment resulted in grouping $1 / 25 L_{20}$ and $1 / 5 L_{20}$ samples into the same cluster. This observation can be explained by the difference of clinical doses of the drugs. It is known that cerivastatin has more potent HMG-CoA reductase inhibition effect than other statins (Stein et al., 1997). Based on the HCA results, it seemed that cerivastatin also caused more toxicity than other two statins at $1 / 25 \mathrm{LC}_{20}$ concentration.

\section{Isolation of differentially expressed genes by statin treatment}

Genes whose expressions are highly modulated (more than two fold) by statin treatments compared with control were isolated from each drug and dose conditions after ANOVA test described in the previous section. Table 1 summarized numbers of genes showed more two expression level changes in treatment group. In brief, cerivastatin seemed to cause the most serious ef- fect on gene expression in every dose conditions. Numbers of significantly modulated genes by cerviastatin were greater up to five fold than lovastatin or simvastatin depending on the dose conditions. Since cerivastatin showed very similar expression patterns in 1/25 and $1 / 5 \quad L_{20}$ doses and relatively small numbers of DEG (342 and 409 genes), both dose groups were com-

Table 1. Numbers of significantly modulated probes by statin treatments in rat primary hepatocytes

\begin{tabular}{|c|c|c|c|c|c|}
\hline \multicolumn{6}{|c|}{ Dose } \\
\hline Drug & & EC & $1 / 25 \quad L^{20}$ & $1 / 5 \quad L^{20}$ & $\mathrm{LC}_{20}$ \\
\hline \multirow[t]{3}{*}{ Cerivastatin } & Up & 92 & 148 & 169 & 728 \\
\hline & Down & 27 & 194 & 240 & 1,071 \\
\hline & Total & 119 & 342 & 409 & 1,799 \\
\hline \multirow[t]{3}{*}{ Simvastatin } & Up & 32 & 36 & 124 & 363 \\
\hline & Down & 1 & 1 & 30 & 468 \\
\hline & Total & 33 & 37 & 154 & 831 \\
\hline \multirow[t]{3}{*}{ Lovastatin } & Up & 36 & 45 & 51 & 191 \\
\hline & Down & 8 & 24 & 28 & 240 \\
\hline & Total & 44 & 69 & 79 & 431 \\
\hline
\end{tabular}

The cut-off for significance $p$-value $<0.05$ (ANOVA test) and change of probe intensity $>2$.

A)

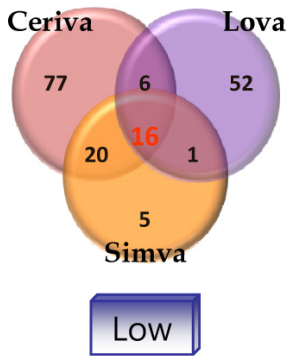

B)

PCA analysis

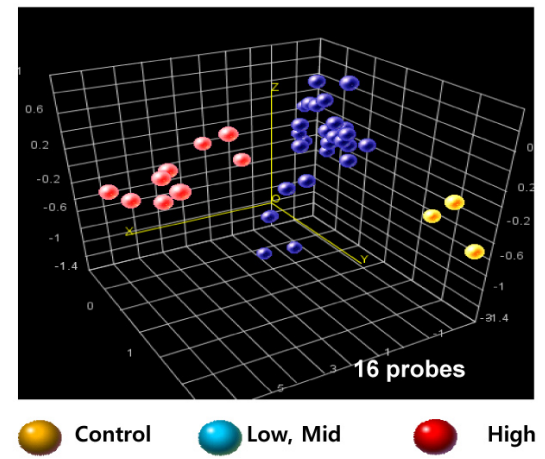

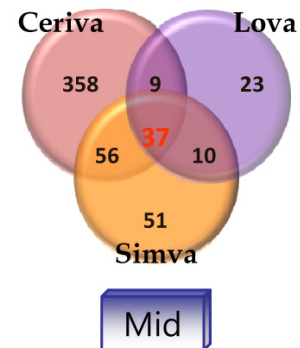

Mid

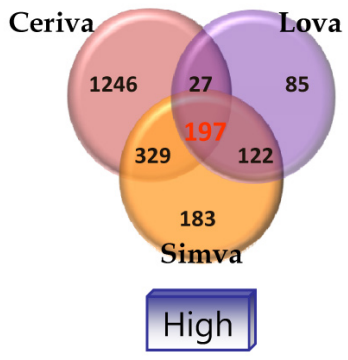

Fig. 3. Venn Diagram analysis and PCA analysis of commonly modulated probes by statin treatment. Probes commonly modulated by statin treatments in each dose group were isolated by Venn Diagram analysis (A). PCA of sample groups were performed by combined probe sets came from common probes of each dose group (B). PCA showed that the common probe sets classified statin treated samples into three clusters according based on the drug doses applied to the samples. 
bined into a single group (Mid) in the following common probe isolation, KEGG and GO analysis. In the contrary, lovastatin and simvastatin revealed similar expression patterns and small DEG numbers at EC and 1/25 $\mathrm{LC}_{20}$ dose groups and the two groups were combined into a single group (Low) as well (Fig. 2 and Table 1).

\section{Isolation of Common genes modulated by three statins}

As mentioned in previous section, samples were grouped as low, mid and high depending on doses and drugs. Using Venn diagram analysis, commonly modulated genes by the drugs at each dose were isolated. As seen Fig. 3, 16, 37 and 197 genes were commonly presented in low, mid and high-dose treatment respectively. According to KEGG pathway analysis (Kanehisa and Goto, 2000) of these commonly modulated genes, all three drugs modulated genes related to statin efficacy, such as biosynthesis of steroids and fatty acid metabolism, at low and mid dose groups (Table 2). But in high dose treatment, the drugs modulated genes related to cell cycle regulation and immune response as well as genes in fatty acid metabolism. This observation suggested that the drugs provoked considerable cytotoxicity in rat primary hepatocytes at high dose (Table 2). Principal component analysis (PCA) of these commonly modulated genes (219 in total) classified samples into three well defined groups such as control, a group mixed with low and mid doses and a group of high doses. Based on these results, it seemed obvious that statin treatment caused toxic effect and distinctive gene modulation only at high dose administration (Fig. 3).

\section{Gene Ontology analysis of DEG by statin treat- ment}

To further investigate which biological process were affected by statin treatment, gene ontology (GO) analysis was performed by using PANTHER program (Protein ANalysis THrough Evolutionary Relationships Classification System, www.pantherdb.org, Mi, et al., 2005) and enriched GO terms by statin treatment were isolated

Table 2. KEGG pathways and the numbers of commonly modulated probes at different doses

\begin{tabular}{|c|c|c|c|c|c|c|c|c|}
\hline \multicolumn{3}{|l|}{ Low } & \multicolumn{3}{|l|}{ Mid } & \multicolumn{3}{|l|}{ High } \\
\hline Pathway & $\begin{array}{c}\text { Probe } \\
\text { No. }\end{array}$ & p-value* & Pathway & $\begin{array}{l}\text { Probe } \\
\text { No. }\end{array}$ & p-value* & Pathway & $\begin{array}{l}\text { Probe } \\
\text { No. }\end{array}$ & $p$-value ${ }^{\star}$ \\
\hline Gap junction & 2 & 0.0088 & $\begin{array}{l}\text { Biosynthesis of unsaturated } \\
\text { fatty acids }\end{array}$ & 3 & 0.000082 & PPAR signaling pathway & 6 & 0.000091 \\
\hline Biosynthesis of steroids & 1 & 0.037 & PPAR signaling pathway & 3 & 0.0022 & $\begin{array}{l}\text { Biosynthesis of unsaturated } \\
\text { fatty acids }\end{array}$ & 3 & 0.0019 \\
\hline $\begin{array}{l}\text { Biosynthesis of } \\
\text { unsaturated fatty acids }\end{array}$ & 1 & 0.039 & Biosynthesis of steroids & 2 & 0.0031 & $\begin{array}{l}\text { Glycine, serine and threonine } \\
\text { metabolism }\end{array}$ & 3 & 0.011 \\
\hline Butanoate metabolism & 1 & 0.057 & Fatty acid metabolism & 2 & 0.01 & Selenoamino acid metabolism & 2 & 0.014 \\
\hline $\mathrm{N}$-Glycan biosynthesis & 1 & 0.068 & $\begin{array}{l}\text { Metabolism of xenobiotics by } \\
\text { cytochrome P450 }\end{array}$ & 2 & 0.013 & Methionine metabolism & 2 & 0.024 \\
\hline Taste transduction & 1 & 0.068 & Retinol metabolism & 2 & 0.018 & $\begin{array}{l}\text { Complement and coagulation } \\
\text { cascades }\end{array}$ & 3 & 0.038 \\
\hline \multirow{8}{*}{$\begin{array}{l}\text { Hedgehog signaling } \\
\text { pathway }\end{array}$} & 1 & 0.084 & Nucleotide sugars metabolism & 1 & 0.019 & Calcium signaling pathway & 5 & 0.048 \\
\hline & & & $\begin{array}{l}\text { Drug metabolism - cytochrome } \\
\text { P450 }\end{array}$ & 2 & 0.02 & ECM-receptor interaction & 3 & 0.05 \\
\hline & & & $\begin{array}{l}\text { Gamma-Hexachlorocyclohexane } \\
\text { degradation }\end{array}$ & 1 & 0.053 & Focal adhesion & 5 & 0.052 \\
\hline & & & Galactose metabolism & 1 & 0.078 & TGF-beta signaling pathway & 3 & 0.058 \\
\hline & & & Glutamate metabolism & 1 & 0.082 & Small cell lung cancer & 3 & 0.064 \\
\hline & & & Linoleic acid metabolism & 1 & 0.093 & Aminoacyl-tRNA biosynthesis & 2 & 0.071 \\
\hline & & & Aminosugars metabolism & 1 & 0.1 & N-Glycan biosynthesis & 2 & 0.074 \\
\hline & & & & & & $\begin{array}{l}\text { Synthesis and degradation of } \\
\text { ketone bodies }\end{array}$ & 1 & 0.084 \\
\hline
\end{tabular}

*p-value by Fisher's exact test. 
(Fig. 4). Gene Ontology analysis also showed that the most enriched GO terms by statin treatments belonged to cellular metabolic process and response to stress. In particular, GO terms related to lipid, fatty acid and steroid metabolism were highly over-represented in most treated groups representing efficacy of statins in rat primary hepatocytes. It was also remarkable that GO terms such as immunity and defense and blood clotting were highly over-represented in high dose groups especially in cerivastatin treatment groups. This observation

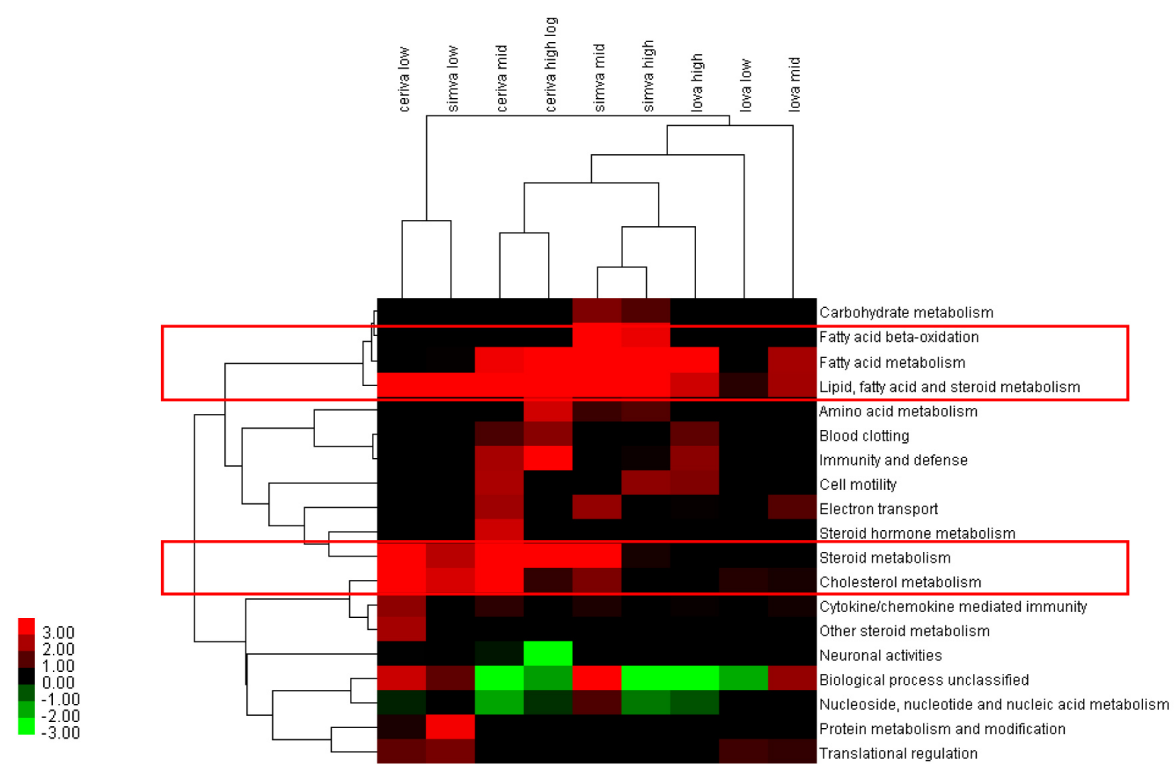

over/under represented biological processes $(p<0.05)$ by statins based on DEG list $(>2$-fold, $p<0.05$ )
Fig. 4. Enriched GO biological process terms by statin treatments using differentially expressed probes in rat primary hepatocytes $(p<0.05)$. GO analysis of differentially expressed probes $(p<$ 0.05 , fold change $>2$ ) were used for enrichment analysis of GO biological process terms provided by PANTHER website. Biological processes showed significant enrichment by statin treatments were used to draw heatmap $(p<0.05)$.

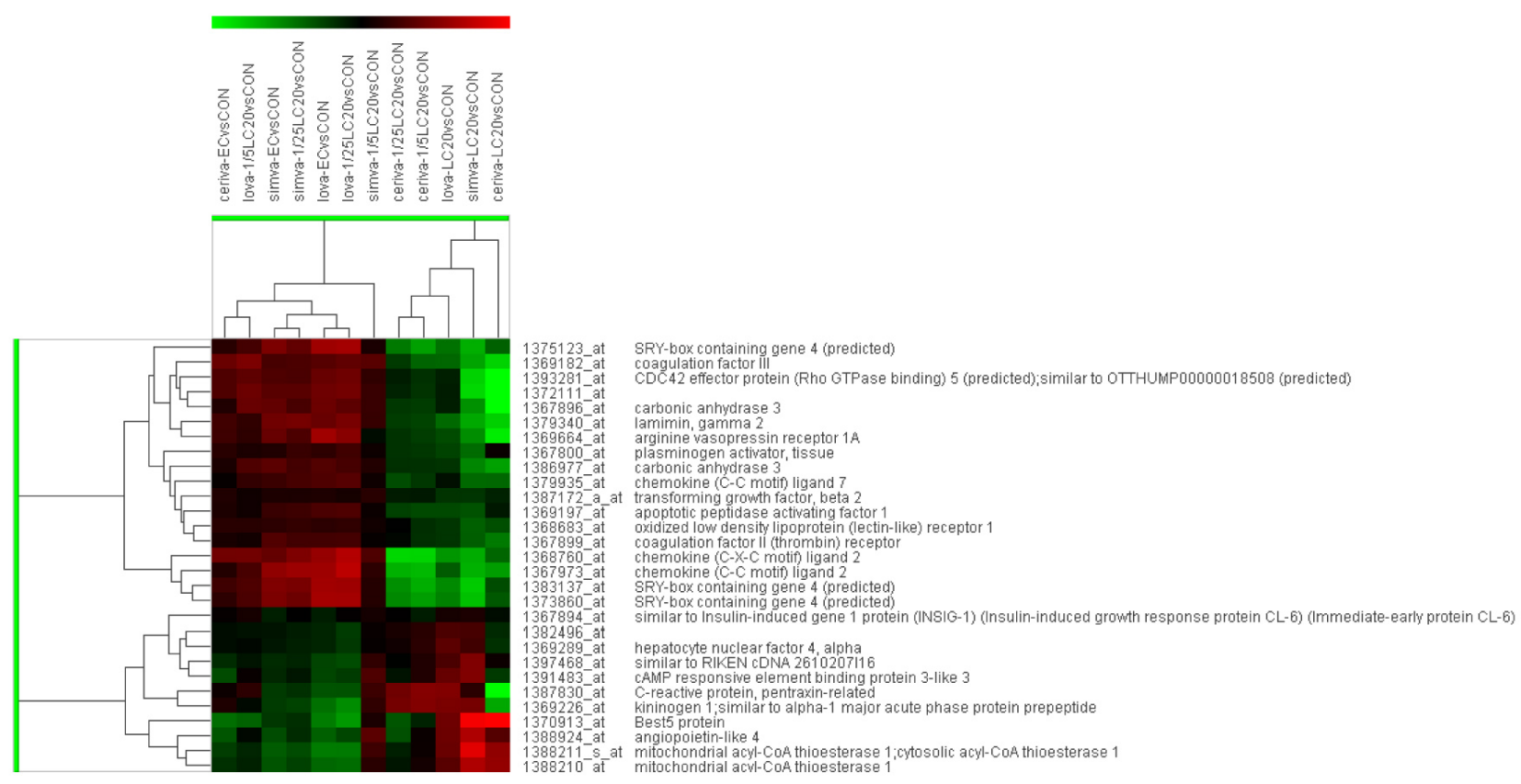

Fig. 5. Hierarchical clustering of commonly modulated genes related to response to stress. Expression patterns of genes related to response to stress were clustered by HCA. Known annotations of corresponding genes were given to each probe. 
suggested that genes in these GO terms had certain relevance to cytotoxicity of the drugs.

\section{Isolation of indicator genes for statin-induced cytotoxicity}

Based on what we observed from DEG analysis and GO analysis of DEG, we tried to isolate genes which may serve as indicator genes for statin-induced cytotoxicity in rat primary hepatocytes. For this purpose, genes which were significantly modulated by all three statins and belonged to response to stress were isolated as putative markers for statin-induced cytotoxicity. 29 probes fit to these criteria were isolated and subjected to hierarchical clustering (Fig. 5). HCA showed a very clear clustering of genes and samples into two groups. First cluster was composed of low dose treatments of each drug such as EC and 1/25 $\mathrm{LC}_{20}$ dose groups except for $1 / 5 L_{20}$ group of lovastatin treatment. Second cluster was mainly composed of high dose samples such as $1 / 5 L_{20}$ and $L_{2}$ groups except $1 / 25 L_{20}$ of cerivastatin. The last cluster was composed of two high dose samples of cerivastatin and simvastatin. Based on the expression pattern of selected genes, cerivastatin seemed to have greater effect on expression of cytotoxicity related genes than other two statins. On the contrary, lovastatin seemed to have the least effect on the expression of cytotxocity related genes. Taken together presented results and previous reports, the isolated 29 probes may serve as indicator genes of statin-induced cytotoxicity in rat primary hepatocytes.

\section{PCR verification of expression level of the puta- tive indicator genes}

Since understanding the gene level mechanism of statin-caused hepatotoxicity was main purpose of this study, selected 29 probes were further studied. First, bi-
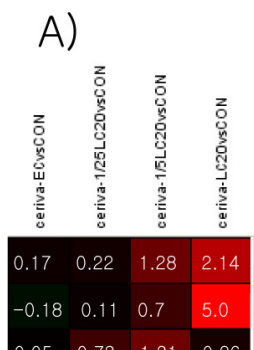

\begin{tabular}{|l|l|l|l|}
0.05 & 0.73 & 1.31 & 0.26 \\
\hline
\end{tabular}

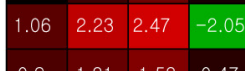

\begin{tabular}{|l|l|l|l|}
\hline 0.9 & 1.21 & 1.52 & 0.47 \\
\hline 1.18 & 2.21 & 2.37 & -1.19 \\
\hline
\end{tabular}

\begin{tabular}{|l|l|l|l|}
\hline 1.18 & 2.21 & 2.37 & -1.13 \\
\hline
\end{tabular}

\begin{tabular}{|l|l|l|l|}
\hline 0.76 & 1.09 & 1.57 & 3.1 \\
\hline
\end{tabular}

\begin{tabular}{|c|c|c|c|}
\hline 0.43 & 0.9 & 1.31 & 1.07 \\
\hline-0.52 & -1.61 & -1.72 & -1.14 \\
\hline
\end{tabular}

\begin{tabular}{|l|l|l|l|}
-0.52 & -1.61 & -1.72 & -1. \\
\hline-0.67 & -1.98 & -2.11 & -4.16 \\
\hline
\end{tabular}

\begin{tabular}{l|l|l|l}
-0.67 & -1.98 & -2.11 & -4.16 \\
\hline-1.54 & -4.02 & -4.11 & -3.79 \\
\hline-1.16 & -2.11 & -1.84 & -2.43 \\
\hline
\end{tabular}

\begin{tabular}{l|l|l|l|}
-1.16 & -2.11 & -1.84 & -2.43 \\
\hline 0.18 & -1.05 & -1.28 & -3.76 \\
\hline
\end{tabular}

\begin{tabular}{l|l|l|l}
0.18 & -1.05 & -1.28 & -3.76 \\
\hline 0.19 & -4.04 & -4.0 & -2.7 \\
\hline 0.68 & -1.03 & -1.51 & -2.4
\end{tabular}

\begin{tabular}{lll|l|l}
-0.68 & -1.03 & -1.51 & -2.4 \\
\hline 0.28 & -1.63 & -2.14 & -3.44
\end{tabular}

\begin{tabular}{|l|l|l|l|}
\hline-0.43 & -1.88 & -1.96 & -3.6 \\
\hline
\end{tabular}

\begin{tabular}{lllll}
-0.47 & -0.95 & -1.48 & -1.86 \\
\hline
\end{tabular}

\begin{tabular}{lllll}
0.08 & -0.97 & -1.02 & -0.29 \\
\hline
\end{tabular}

\begin{tabular}{|l|l|l|l|}
\hline-0.9 & -3.04 & -3.4 & -2.5 \\
\hline-0.36 & -1.06 & -1.02 & -1.15 \\
\hline
\end{tabular}

${ }_{30}$
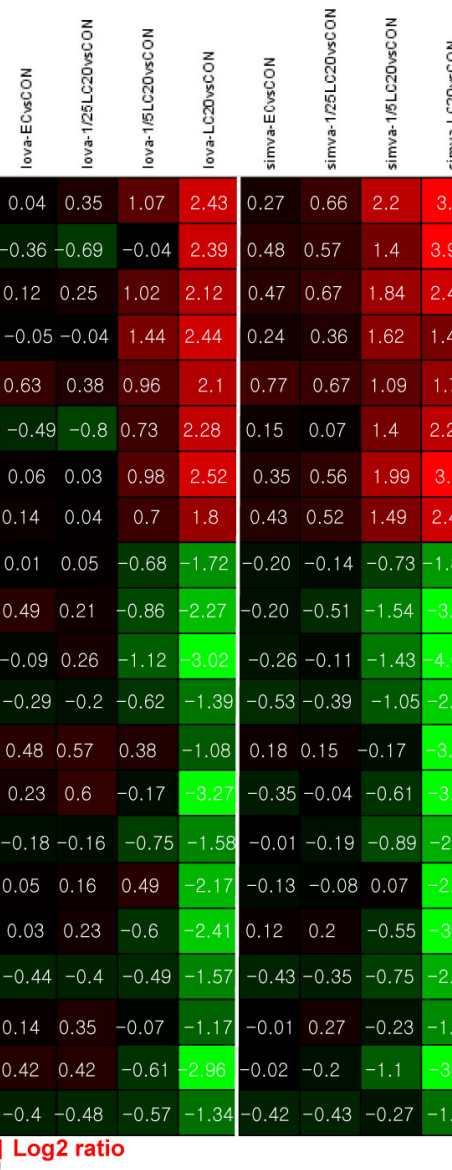

B)

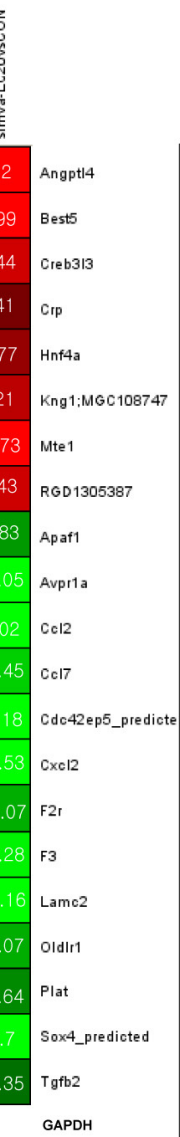

Lovastatin

Simvastatin

$\begin{array}{lllllllllll}\text { C EC } & 1 / 25 & 1 / 5 & \text { LC20 EC } & 1 / 25 & 1 / 5 & \text { LC20 EC } & 1 / 25 & 1 / 5 & \text { LC20 }\end{array}$

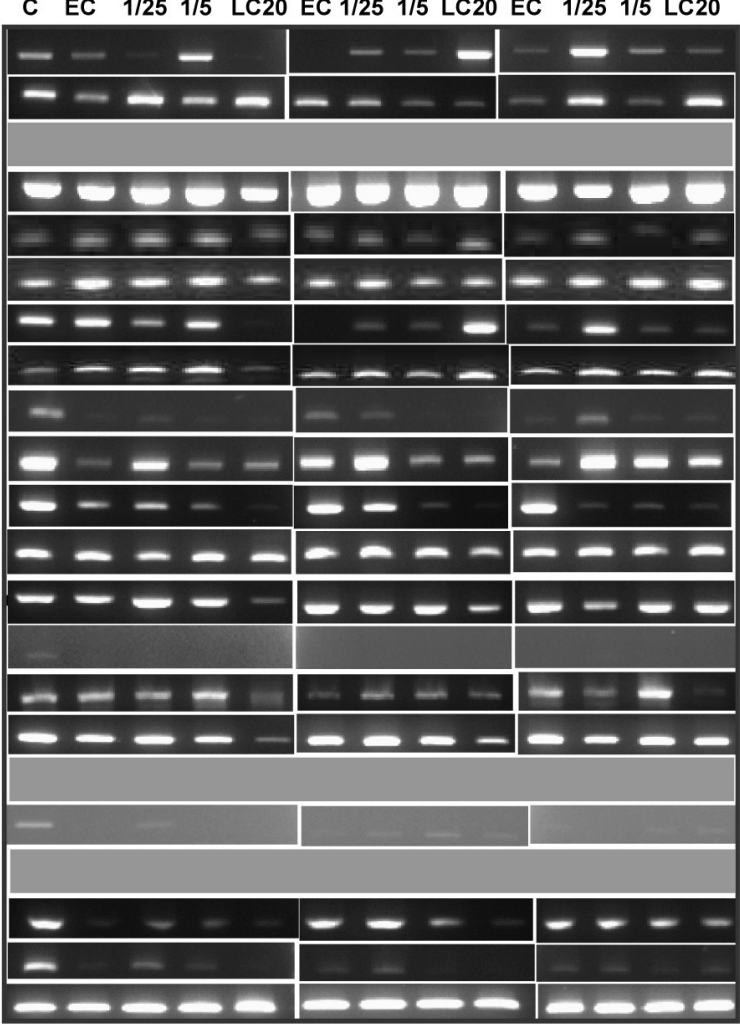

Fig. 6. Expression patterns of putative indicator genes obtained by microarray and by RT-PCR. Expressions of putative indicator genes were shown in $\log _{2}$ scale $(A)$ and by RT-PCR (B). 
ological function of each probe was watched for and corresponding genes were isolated. From 29 probes isolated as stress markers, we were able to identify 21 genes whose functions are annotated (Fig. 6). It was remarked that many genes are known to have their involvements in inflammatory or coagulation response such as Ccl2, Ccl7, Cxcl2, F2r, F3 and Crp among annotated genes (Bharadwaj et al., 1999; Hsu et al., 2006; Johnston et al., 1999). This observation concurred with previously reported clinical effects of statins on inflammation or coagulation (Dols et al., 2009, Maitlandvan der Zee et al., 2009). This implied the involvement of these biological pathways in the observed cytotoxic effect of statins on rat hepatocytes.

Genes involved in cell proliferation were also found from the probe set, for example Cdc42ep5 predicted and Tgfbeta2. Beside genes involved in inflammation and proliferation, a liver specific gene, Hnf4alpha was isolated. Hnf4alpa is known as a liver specific transcription factor and reported to have interaction with SMAD3/4 protein in TGFbeta pathway (Zannis et al., 2001). Hnf4alpha is also reported to have implication in ER stress induced acute phase response in liver (Luebke-Wheeler et al., 2008).

Expression of annotated 21 genes was further examined by RT-PCR to confirm microarray results (Fig. 6). As seen in Fig. 6, some of them (Creb3I3, Lamc2, Flat and Oldlr1) did not produce any detectable PCR products but still most of them showed certain concordance to microarray results. In particular, genes whose functions are closely related to liver toxicity such as Crp, Hnf4alpha and TGFbeta2 showed very good concordance between microarray and RT-PCR results.

In the presented study, we employed gene expression profiling techniques and bioinformatic analyses to find a set of genes which may serve as indicators for statin-induced liver toxicity. Since statins are mostly widely used drugs in the world to treat pandemic hyperchoresterolemia and have been reported recurrently to have liver toxicity in clinical uses, finding genes which could explain underlying mechanism and serve as indicator is of most interest in drug development and regulation field. By combining an in vitro model system and microarray techniques, we were able to isolate genes whose expressions were commonly modulated by statins and which also had biological functions related to response to stress. It is still too early to say that the genes listed in this work could serve as biomarkers for statin-induced liver toxicity but they demonstrated the potential of gene expression analysis for understanding and predicting certain effects of drugs such as statin-induced cytotoxicity in rat primary hepatocytes. To further validate current findings and make applicable to clinical purpose, it will necessary to compare biomarkers from rat primary hepatocytes with those from human primary hepatocytes or those from human liver tissues (Hart et al., 2010).

\section{Acknowledgements}

This work was supported by a grant (08171kfda501) from the Korea Food \& Drug Administration.

\section{References}

Ahn, J., Jeong, K.J., Ko, M., Shin, H.J., Chung, H.J., and Jeong, H. (2009). High-concentration Epigallocatechin gallate treatment causes endoplasmic reticulum stressmediated cell death in HepG2 cells. Genomics inform. 7, 97-106.

Baycol website. Dear healthcare Letter Baycoll pull.jpg http:// www.baycol.com/.

Bharadwaj, D., Stein, M.P., Volzer, M., Mold, C., and Du Clos, T.W. (1999). The major receptor for C-reactive protein on leukocytes is fcgamma receptor. J. Exp. Med. 16, 585-590.

Bolego, C., Baetta, R., Bellosta, S., Corsini, A., and Paoletti, R. (2002). Safety Considerations for statins. Curr. Opin. Lipidol. 13, 637-644.

Chalasani, N. (2005). Statins and Hepatotoxicity: Focus on patients with fatty liver. Hepatology 41, 690-695.

Dold, S., Laschke, M.W., Lavasani, S., Menger, M.D., Jeppsson, B., and Thorlacius, H. (2009). Simvastatin protects against cholestasis-induced liver injury. $\mathrm{Br} . \mathrm{J}$. Pharmacol. 156, 466-474.

Hamelin, B.A., and Turgeon, J. (1998). Hydrophilicity/lipophilicity: relevance fro the pharmacology and clinical effects of HMG-CoA reductase inhibitors. Trends Pharmacol. Sci. 19, 26-37.

Hart, S.N., Li, Y., Nakamoto, K., Subileau, E.A., Steen, D., and Zhong, X.B. (2010). A comparison of whole genome gene expression profiles of HepaRG cells and HepG2 cells to primary human hepatocytes and human liver tissues. Drug Metab. Dispos. March 12, [Epub ahead of print]

Heart protection study collaborative group. (2002). MRC/BHF Heart Protection Study of cholesterol lowering with simvastatin in 20536 high-risk individuals: a randomized placebo-controlled trial. Lancet. 360, 7-22.

Hsu, C.C., Lin, C.C., Liao, T.S., and Yin, M.C. (2006). Protective effect of s-allyl cysteine and s-propyl cysteine on acetaminophen-induced hepatotoxicity in mice. Food Chem. Toxicol. 44, 393-397.

Johnston, B., Burns, A.R., Suematsu, M., Issekutz, T.B., Woodman, R.C., and Kubes, P. (1999). Chronic inflammation upregulates chemokine receptors and induces neutrophil migration to monocyte chemoattractant protein-1. J. Clin. Invest. 10, 1269-1276.

Kanehisa, M., and Goto, S. (2000). KEGG: Kyoto Encyclopedia of Genes and Genomes. Nucl. Acids Res. 
$28,27-30$.

Luebke-Wheeler, J., Zhang, K., Battle, M., Si-Tayeb, K., Garrison, W., Chhinder, S., Li, J., Kaufman, R.J., and Duncan, S.A. (2008). HNF4 $\alpha$ is implicated in ER stress induced acute phase response by regulating expression of $\mathrm{CrebH}$. Hepatology 48, 1242-1250.

Maddrey, W.C. (2005). Drug-induced hepatotoxicity. J. Clin. Gastroenterol. 39, S83-S89.

Maitland-van der Zee, A.H., Peters, B.J., Lynch, A.I., Boerwinkle, E., Arnett, D.K., Cheng, S., Davis, B.R., Leiendecker-Foster, C., Ford, C.E., and Eckfeldt, J.H. (2009). The effect of nine common polymorphisms in coagulation factor genes (F2, F5, F7, F12 and F13) on the effectiveness of statins: the GenHAT study. Pharmacogenet. Genomics 19, 338-344.

Mi, H., Lazareva-Ulitsky, B., Loo, R., Kejariwal, A., Vandergriff, J., Rabkin, S., Guo, N., Muruganujan, A., Doremieux, O., Campbell, M.J., Kitano, H., and Thomas, P.D. (2005). The PANTHER database of protein families, subfamilies, functions and pathways. Nucl. Acids Res. 33, D284-D288.
Stein, E., Sprecher, D., Allenby, K.S., Tosiello, R.L., Whalen, E., and Ripa, S.R. (1997). Cerivastatin, a new potent synthetic HMG-CoA reductase inhibitor: Effect of $0.2 \mathrm{mg}$ daily in subjects with primary hypercholesterolemia. J. Cardiovasc. Pharmacol. Ther. 2, 7-16.

Vasudevan, A.R., Hamirani, Y.S., and Jones, P.H. (2005). Safety of statins: effect on muscle and the liver. Cleve. Clin. J. Med. 72, 990-993.

Wilmshurst, P. (2002). Heart Protection Study. Lancet. 2003 Feb 8;361, 528-529 author reply 529-530.

Yamazaki, H., Suzuki, M., Alki, T., Morikawa, S., Maejima, T., and Sato, F. (2006). Influence of 3-hydroxy-3-methylglutaryl coenzyme a reductase inhibitors of ubiquinone level in rat skeletal muscle and heart: relationship to cytotoxicity and inhibitory activity for cholesterol synthesis in human skeletal muscle cell. J. Atheroscler. Thromb. 13, 295-307.

Zannis, V.I., Kan, H.Y., Kritis, A., Zanni, E., and Kardassis, D. (2001) .Transcriptional regulation of the human apolipoprotein genes. Front. Biosci. 1, 456-504. 